\title{
Protective effect of lutein on oxidative stress damage caused by acute PM2.5 exposure in rats
}

\author{
Ximin Chen, Yuexiao Chen, Yuan Chen, Xin Wang, Mei He \\ Beijing Institute of Nutritional Sources, Beijing, China \\ Contributions: (I) Conception and design: X Chen, X Wang; (II) Administrative support: Y Chen, M He; (III) Provision of study materials or patients: \\ Y Chen, X Wang; (IV) Collection and assembly of data: X Chen; (V) Data analysis and interpretation: X Chen; (VI) Manuscript writing: All authors; \\ (VII) Final approval of manuscript: All authors. \\ Correspondence to: Xin Wang; Mei He. Beijing Institute of Nutritional Sources, Beijing, China. Email: wangxin@bonnysci.com; hemei2003@163.com.
}

Background: Particulate matter (PM) is the sum of organic and inorganic substances suspended in air. Particles with a diameter equal to or smaller than $2.5 \mu \mathrm{m}$ (PM2.5) can induce extensive oxidative stress damage in lung tissues. Lutein can prevent oxidative stress and the production of pro-inflammatory cytokines. However, its role in lung injury induced by PM2.5 is still unclear. This study used lutein to intervene in lung injury induced by PM2.5 in rats to confirm the protective function of lutein in PM2.5induced lung injury.

Methods: A total of 80 rats were divided into five groups according to body weight (bw). Rats in the blank control group and the PM2.5 exposure group were treated with normal saline. The low-, medium-, and high-dose lutein groups were treated with 12.5, 25.0, and $50.0 \mathrm{mg} / \mathrm{kg} \cdot \mathrm{bw}$ lutein continuously for 28 days. In addition, on days 22-28, $10 \mathrm{mg} / \mathrm{kg}$ PM2.5 was introduced by intratracheal instillation into the PM2.5 exposure group and all lutein dose groups continuously for 7 days to establish PM2.5 injury models. Lung tissues were collected and subjected to hematoxylin-eosin (HE) staining to observe histomorphological changes. Bronchoalveolar lavage fluid (BALF) was collected to detect the expression of cell damage indicators including lactate dehydrogenase (LDH), acidic phosphatase (ACP), alkaline phosphatase (AKP), interleukin-6 (IL-6), interleukin-8 (IL-8), and tumor necrosis factor-alpha (TNF- $\alpha$ ). In addition, the levels of serum oxidative stress indicators in rats, including methane dicarboxylic aldehyde (MDA), superoxide dismutase (SOD), glutathione (GSH), nitric oxide synthase (NOS), and 8-hydroxy-2'-deoxyguanosine (8-OHdG), as well as cytokines, including IL-6, IL-8, and TNF- $\alpha$, were analyzed.

Results: Lung tissues of rats after PM2.5 exposure exhibited different degrees of pathological changes. In serum, oxidative damage indicators MDA, NOS, and 8-OHdG and inflammatory cytokines IL-6, IL-8, and TNF- $\alpha$ significantly increased whereas GSH levels significantly decreased $(\mathrm{P}<0.05)$. In BALF, cell damage indicators ACP and AKP and inflammatory cytokines IL-6, IL-8, and TNF- $\alpha$ all increased $(\mathrm{P}<0.05)$. After lutein intervention, oxidative damage and cellular inflammatory cytokines in serum and BALF all significantly decreased $(\mathrm{P}<0.05)$ whereas serum $\mathrm{GSH}$ levels increased $(\mathrm{P}<0.05)$.

Conclusions: PM2.5 caused significant oxidative damage and inflammatory injury in rat lungs. Lutein alleviated these effects, playing a protective role in lung tissues.

Keywords: Lutein; PM2.5; rats; lung injury; antagonistic effect

Submitted Apr 16, 2020. Accepted for publication Jul 04, 2020.

doi: 10.21037/apm-20-1138

View this article at: http://dx.doi.org/10.21037/apm-20-1138 


\section{Introduction}

Particulate matter (PM2.5) refers to a particle with an aerodynamic equivalent diameter $\leq 2.5 \mu \mathrm{m}$ in ambient air and is an important component of particles in ambient air. Cell experiments have indicated that PM2.5 has toxic effects on cells (1). It can induce cells to produce active oxygen, resulting in oxidative stress reactions and, eventually, DNA damage (2). Animal experiments have indicated that PM2.5 exposure by intratracheal instillation can induce morphological changes and oxidative damage in lung tissues of mice (3). PM2.5 is closely associated with health. It is the top risk factor responsible for reduced longevity in some areas (4). Epidemiological surveys have shown that $40.3 \%$ of stroke deaths, $26.8 \%$ of deaths from local ischemic heart disease, and $23.9 \%$ of lung cancer deaths are associated with high PM2.5 concentrations (5). Molecular studies shows that PM2.5 caused a reduction of mitochondrial markers in mitochondrial dysfunction, including peroxisome proliferator-activated receptorycoactivator-1 (PGC-1), Nuclear respiratory factor-1 (Nrf-1), mitochondrial transcription factor A (TFAM), which suggest that energy metabolic disorders and mitochondrial dysfunction may be the important contributors to pulmonary injuries in response to PM2.5 exposure. Lutein in humans derives from dietary intake because it cannot be synthesized in the body, and it has a relatively high content in dark green vegetables (6). As a food ingredient, it was approved as a new resource food in the No. 12 document in 2008 announced by the former Ministry of Health. As a natural antioxidant, it can inactivate singlet oxygen through physical or chemical quenching effects, capture hydroxyl radicals, bind to lipids to inhibit and prevent lipid oxidation, and reduce free radical damage to biomembranes (7). Previous lutein studies have mainly focused on its protective effect on the retina $(8,9)$. It has been shown that lutein can significantly improve light-induced retinal oxidative damage (10). The concentration of lutein in lung tissue is 0.10 $2.30 \mu \mathrm{mol} / \mathrm{L}$ (11). However, its role in the respiratory tract, especially during injury, is rarely reported. Therefore, this study observed the damaging effect of PM2.5 exposure in rat lung and the effect of lutein in antagonizing this damage in order to investigate the protective effect of lutein in respiratory tract injury caused by PM2.5 in rats.

We present the following article in accordance with the ARRIVE reporting checklist (available at http://dx.doi. org/10.21037/apm-20-1138).

\section{Methods}

\section{Main instruments and reagents}

The TH-1000C Large Flow Air Particle Sampler and PM2.5 selector were from Wuhan Tianhong Instrument Co., Ltd. The DS-3510DTH ultrasonic cleaner was from Shanghai SXSONIC Instrument Co. Ltd. The XS-M precision balance was from Mettler-Toledo (Switzerland). The TDL-5 centrifuge was from Shanghai Anting Scientific Instrument Factory. The alpha-2/LDPlus freeze dryer was from Christ (Germany). The $721 \mathrm{G}$ visible spectrophotometer was from Shanghai Precision \& Scientific Instrument Co., Ltd. The BS-400 automatic biochemical analyzer was from Shenzhen Mindray Bio-Medical Electronics Co., Ltd. The DNM-9602G microplate reader was from Perlong Medical Equipment Co., Ltd. (Beijing, China). The TS100m microscope was from Nikon (Japan). The interleukin-6 (IL-6), interleukin-8 (IL-8), and tumor necrosis factor-alpha (TNF- $\alpha$ ) reagent kits were from Beijing Furui Runkang Bio-Technology Co., Ltd. The methane dicarboxylic aldehyde (MDA), superoxide dismutase (SOD), glutathione (GSH), nitric oxide synthase (NOS), 8-hydroxy-2'-deoxyguanosine (8-OHdG), lactate dehydrogenase (LDH), acidic phosphatase (ACP), and alkaline phosphatase (AKP) measurement reagent kits were from Nanjing Jiancheng Bioengineering Institute.

\section{Experimental animals}

A total of 80 specific-pathogen-free (SPF) male SpragueDawley (SD) rats with a body weight of 180-220 g were selected and purchased from Beijing HFK Bioscience Co., Ltd. [SCXK (Beijing) 2014-004]. The rats were housed in the animal laboratory of the Institute of Materia Medica, Chinese Academy of Medical Sciences \& Peking Union Medical College with alternating light and dark and access to food and water ad libitum.

The study was approved by The Animal Care \&Welfare Committee Institute of Materia Medica, Chinese Academy of Medical Sciences \& Peking Union Medical College (ID: 00005303), and is in compliance with its guidelines for the care and use of animals.

\section{Experimental process}

\section{Preparation of PM2.5 samples}

Between September 2016 and February 2017, PM2.5 
samples were continuously collected for 6 months using an air particle sampler (flow $1 \mathrm{~m}^{3} / \mathrm{min}$ ) on the platform outside the Beijing Institute of Nutritional Sources. The glass fiber filter for collecting samples was replaced once every $24 \mathrm{~h}$, and the sample collection height was $5-8 \mathrm{~m}$. The collected glass fiber filters with attached fine particles were cut into $1 \mathrm{~cm} \times 1 \mathrm{~cm}$ pieces, immersed in deionized water, and eluted 3 times (30 min each) using low-temperature ultrasonication in an ultrasonic cleaner. The elution solution was filtered through 6 layers of gauze and centrifuged at 12,000 r/min for $30 \mathrm{~min}$ at $4{ }^{\circ} \mathrm{C}$ to collect the lower suspension. The elution suspension was lyophilized using a vacuum freeze dryer to obtain PM2.5 samples after water was completely vaporized. Samples were sealed and stored in a $-20{ }^{\circ} \mathrm{C}$ freezer. Before use, samples were thawed at room temperature. The samples were then weighed and prepared as suspensions at the required concentration using normal saline. Samples were mixed thoroughly by ultrasonication and stored at $4{ }^{\circ} \mathrm{C}$.

\section{Rat grouping and treatment}

A total of 80 rats were randomly divided into five groups: the normal group, the PM2.5 exposure group, and the low-, medium-, and high-dose lutein groups, with 16 rats in each group. The normal group received normal saline gavage for 28 consecutive days. On days 22-28, the rats received intratracheal instillation of normal saline daily. The PM2.5 group received normal saline gavage for 28 consecutive days. On days $22-28$, the rats received intratracheal instillation of PM2.5 suspension daily. The low-, medium-, and high-dose lutein groups received gavages of 12.5 , 25.0 , and $50.0 \mathrm{mg} / \mathrm{kg} \cdot$ bw lutein for 28 days, respectively. On days 22-28, the rats were exposed to PM2.5 for 7 days using intratracheal instillation of PM2.5 suspension daily. The dose of the gavage was $20 \mathrm{~mL} / \mathrm{kg}$, and the dose of the PM2.5 in the intratracheal instillation was $10 \mathrm{mg} / \mathrm{kg}$ once every day. During the experiment, the general condition of the rats was observed, and their weights were recorded.

\section{Rat processing and sample collection}

Rats were sacrificed within $24 \mathrm{~h}$ of the last exposure. They were anesthetized by intraperitoneal injection of $10 \%$ chloral hydrate, axillary arterial blood samples were collected, and then, the rats were sacrificed. Serum samples were allowed to sit at room temperature for $1 \mathrm{~h}$ and then centrifuged at $3,000 \mathrm{r} / \mathrm{min}$ at $4{ }^{\circ} \mathrm{C}$. The supernatant was collected, aliquoted, and stored in a $-20^{\circ} \mathrm{C}$ freezer. The thoracic cavity was exposed, the left main bronchus was clamped with hemostatic forceps, and a small opening was made. A pulmonary lavage needle was inserted, and the opening was ligated using surgical thread. Phosphatebuffered saline (PBS) solution $(1 \mathrm{~mL})$ was slowly injected into the lung and was then aspirated after the lung was massaged. This step was repeated 3 times. Bronchoalveolar lavage fluid (BALF) was collected and centrifuged at $1,200 \mathrm{r} / \mathrm{min}$ at $4{ }^{\circ} \mathrm{C}$. The supernatant was collected, aliquoted, and stored in a $-20^{\circ} \mathrm{C}$ freezer.

\section{Detection of oxidative damage indicators}

Serum oxidative stress indicators in rats, including MDA, SOD, GSH, NOS, and 8-OHdG, were detected using reagent kits.

\section{Detection of cytokines}

Serum and BALF were collected to measure the levels of IL-6, IL-8, and TNF- $\alpha$ according to the kit instructions.

\section{Detection of cell damage indicators}

BALF was collected to measure the levels of LDH, ACP, and AKP according to the kit instructions.

\section{Staining of pathological sections of lung tissues}

Left lung tissue without lavage was collected and fixed in $10 \%$ formalin for $48 \mathrm{~h}$. Lung tissue from the middle section was collected, embedded in paraffin, and sectioned. The sections were stained with hematoxylin-eosin (HE), and the extent of lung tissue injury and inflammatory cell infiltration was observed under an electron microscope.

\section{Data processing}

All data are expressed as $\mathrm{x} \pm \mathrm{s}$. Data processing was performed using SPSS 18.0 and Origin 2018, including normality tests and variance homogeneity tests. Comparisons of mean values between two groups were performed using the independent-samples $t$-test. Comparisons between different concentration groups and the control group were performed using one-way analysis of variance (ANOVA). $\mathrm{P}<0.05$ indicated that a difference was statistically significant.

\section{Results}

\section{Morphological changes in lung tissues}

Lung tissue injury and the protective effect of lutein pretreatment on rat lung tissues are shown in Figure 1. In 


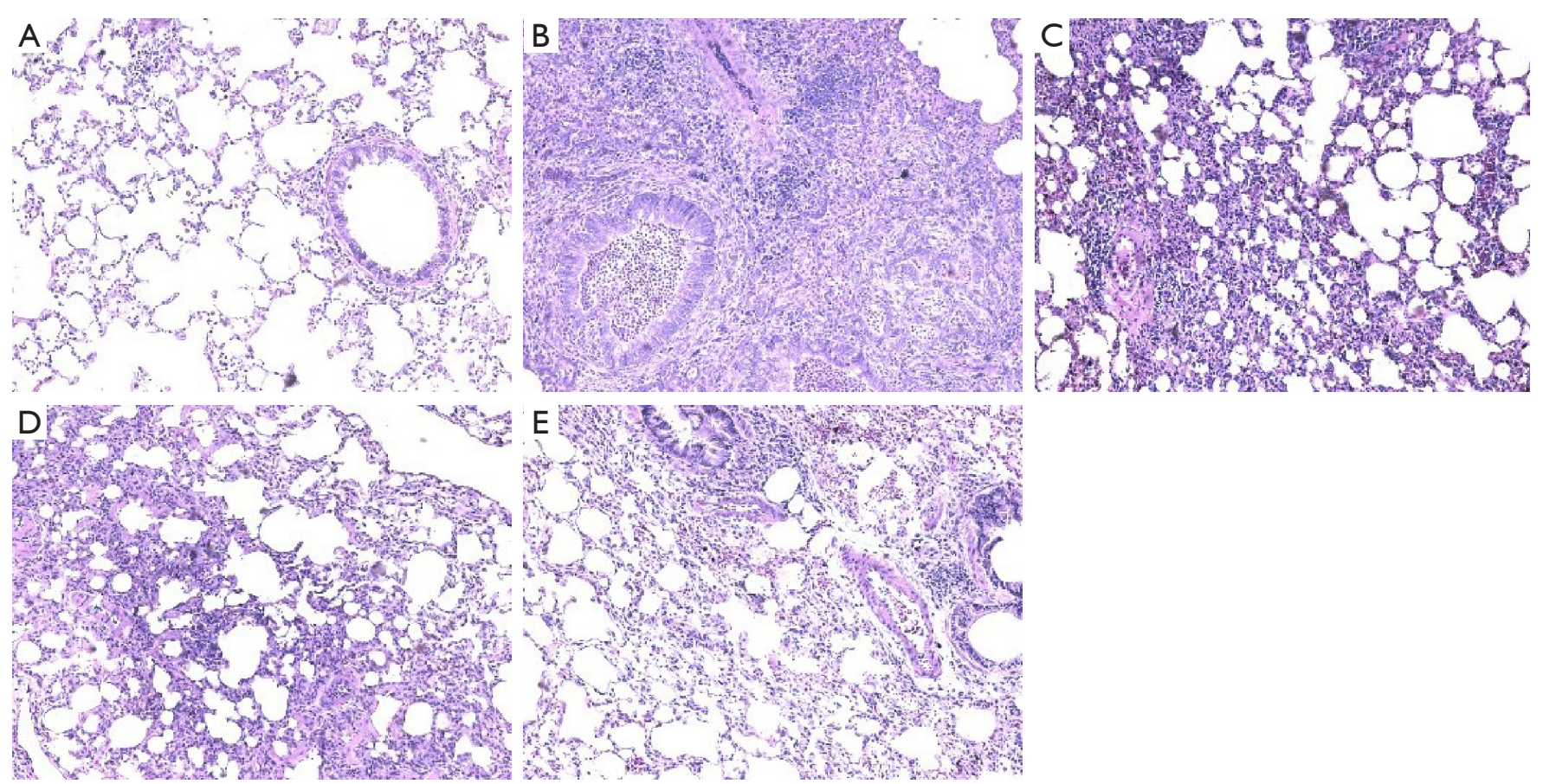

Figure 1 Pathomorphological changes $(\times 200)$. (A,B,C,D,E) Are images from the normal group, PM2.5 exposure group, low-dose group, medium-dose group, and high-dose group, respectively. Stained with hematoxylin and eosin.

the normal group, lung tissues had a normal structure, the bronchial epithelium was intact, and there was no exudate in the lumen. In the PM2.5 exposure group, the lumen was narrow, the tube wall shrank, there was exudate in the lumen, and there was a large amount of inflammatory cell infiltration. Inflammatory cell infiltration in all lutein dose groups decreased.

\section{The oxidative damage effect of PM2.5 and the antagonizing effect of lutein in rats}

The results of the effects of PM2.5 exposure and different doses of lutein intervention on serum MDA, SOD, GSH, NOS, and 8-OHdG in rats are shown in Table 1. After PM2.5 exposure, the levels of serum MDA and NOS in rats were significantly higher than those in the normal group, and the level of GSH was significantly lower than that in the normal group $(\mathrm{P}<0.05)$, indicating that $\mathrm{PM} 2.5$ exposure caused oxidative damage in the lungs of rats. Compared with that in the PM2.5 exposure group, the serum MDA levels in rats in the low-, medium-, and high-dose lutein intervention groups significantly decreased, and the GSH levels significantly increased $(\mathrm{P}<0.05)$, indicating that lutein could help clear MDA produced by oxidative damage in lungs and increase GSH synthesis and secretion. After highdose lutein intervention, the serum NOS level significantly decreased $(\mathrm{P}<0.05)$. Although the serum NOS level showed a decreasing trend after low-dose lutein intervention, the difference was not significant $(\mathrm{P}>0.05)$. Compared with that in the normal group, the serum $8-\mathrm{OHdG}$ level in rats in the exposure group significantly increased $(\mathrm{P}<0.05)$, indicating that this dose of PM2.5 could cause DNA oxidative damage in rat lung tissues. Compared with that in the PM2.5 exposure group, the 8-OHdG levels in the medium- and high-dose lutein groups significantly decreased $(\mathrm{P}<0.05)$; in addition, with the increase in dose, the degree of reduction was higher. These results indicated that lutein may alleviate DNA oxidative damage and reduce PM2.5-induced oxidative damage in the body.

\section{The effect of PM2.5 on cytokines and the antagonizing effect of lutein in rats}

\section{The effect of PM2.5 on IL-6 and the intervention effect of lutein}

IL-6 is produced by various cells, such as macrophages and lymphocytes. It can amplify inflammation during inflammatory reactions (12). After PM2.5 exposure, IL-6 
Table 1 The antagonizing effect of lutein on serum oxidative damage indicators induced by PM2.5

\begin{tabular}{lcccccc}
\hline Group & Quantity $(\mathrm{n})$ & MDA $(\mathrm{nmol} / \mathrm{L})$ & $\mathrm{SOD}(\mathrm{U} / \mathrm{mL})$ & $\mathrm{GSH}(\mu \mathrm{mol} / \mathrm{L})$ & $\mathrm{NOS}(\mathrm{U} / \mathrm{mL})$ & $8-\mathrm{OHdG}(\mathrm{U} / \mathrm{L})$ \\
\hline Normal group & 16 & $8.01 \pm 0.48$ & $561.78 \pm 159.57$ & $65.17 \pm 5.49$ & $16.16 \pm 3.03$ & $19.29 \pm 4.27$ \\
PM2.5 exposure group & 16 & $10.13 \pm 1.01^{*}$ & $576.36 \pm 117.13$ & $46.72 \pm 4.15^{\star}$ & $25.72 \pm 5.66^{*}$ & $49.84 \pm 10.49^{\star}$ \\
Low-dose group & 16 & $7.34 \pm 2.44^{\#}$ & $504.02 \pm 103.68$ & $77.60 \pm 14.31^{\#}$ & $22.19 \pm 7.71^{*}$ & $41.55 \pm 13.39^{\star}$ \\
Medium-dose group & 16 & $7.97 \pm 2.05^{\#}$ & $522.56 \pm 75.14$ & $71.15 \pm 17.70^{\#}$ & $25.28 \pm 5.15^{\star}$ & $37.87 \pm 16.22^{\star^{\#}}$ \\
High-dose group & 16 & $6.88 \pm 1.19^{\#}$ & $511.51 \pm 101.05$ & $78.42 \pm 12.56^{\#}$ & $21.33 \pm 4.98^{\#}$ & $30.91 \pm 16.17^{\star^{\#}}$ \\
\hline
\end{tabular}

*, compared with the normal group, $\mathrm{P}<0.05$; ", compared with the $\mathrm{PM} 2.5$ exposure group, $\mathrm{P}<0.05$.

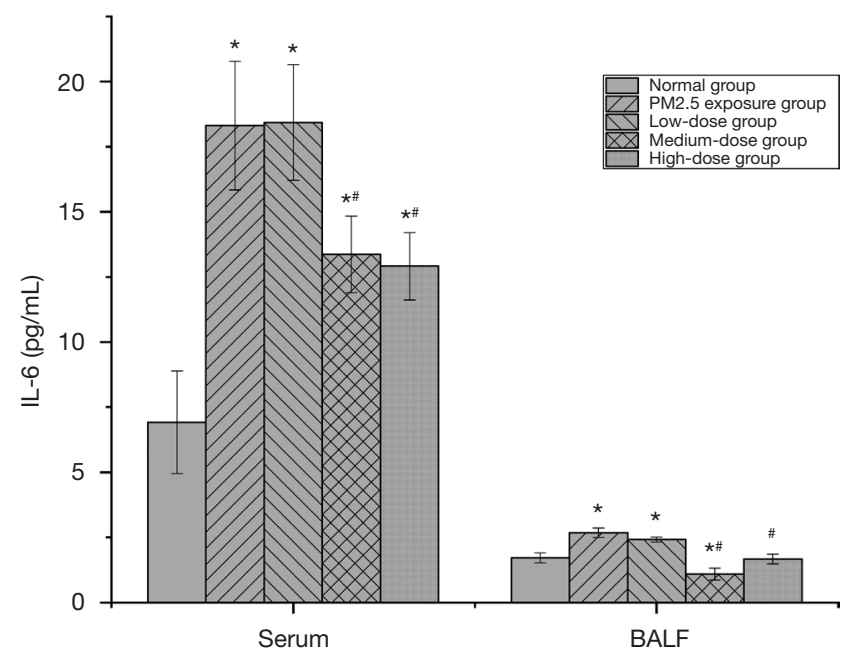

Figure 2 The effect of lutein on IL-6 in serum and BALF. *, compared with the normal group, there was a significant difference $(\mathrm{P}<0.05)$; ", compared with the PM2.5 exposure group, there was a significant difference $(\mathrm{P}<0.05)$.

levels in the serum and BALF of rats both significantly increased $(\mathrm{P}<0.05)$, indicating that persistent $\mathrm{PM} 2.5$ exposure caused cell injury in the lung tissues of rats. After low-dose lutein intervention, IL-6 levels in the serum and BALF of rats did not significantly decrease $(\mathrm{P}>0.05)$. After medium-dose lutein intervention, IL-6 levels in serum and BALF both significantly decreased, indicating that a medium dose of lutein improved cell injury in the lung tissues of rats. When the dose was increased to a high dose, continuous improvement of cellular IL-6 in lung tissues by lutein was not observed (Figure 2).

The effect of PM2.5 on IL-8 and the intervention effect of lutein

IL-8 is an inflammatory cytokine with chemotactic

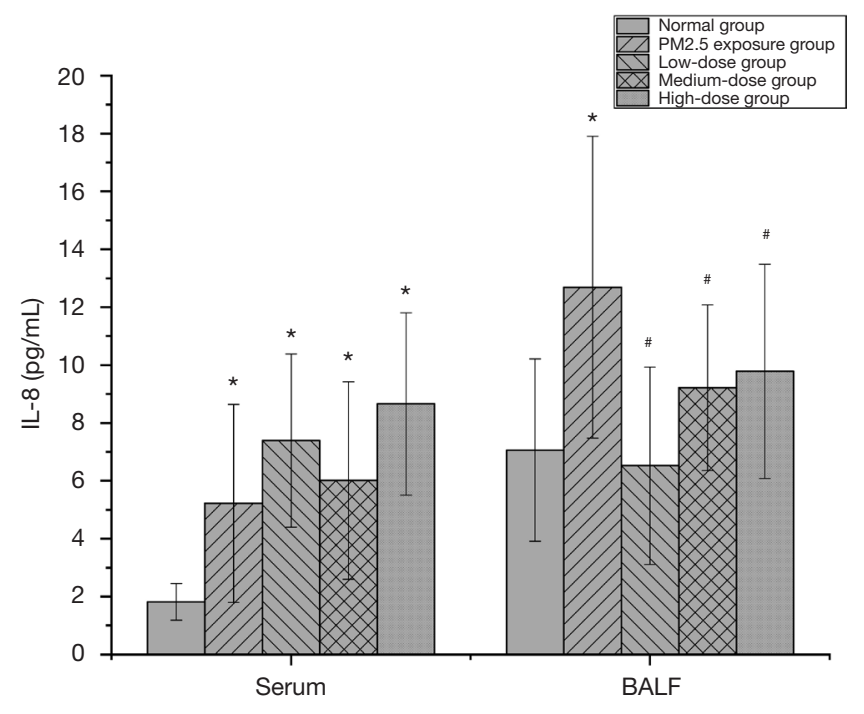

Figure 3 The effect of lutein on IL-8 in serum and BALF. *, compared with the normal group, there was a significant difference $(\mathrm{P}<0.05)$; $^{\text {, }}$ compared with the PM2.5 exposure group, there was a significant difference $(\mathrm{P}<0.05)$.

functions. Its key function is to attract neutrophils to inflammatory sites. Many studies $(13,14)$ have confirmed that IL-8 increases lymphatic metastasis in lung cancer and tumor microvascular density. Furthermore, it is an important indicator for monitoring the treatment effects and evaluating the prognosis of patients with non-small cell lung cancer. IL-8 levels in the serum and BALF of rats were analyzed, and the results are shown in Figure 3. Serum IL-8 levels in rats in the PM2.5 exposure group and all lutein dose groups significantly increased $(\mathrm{P}<0.05)$, indicating that PM2.5 significantly promoted IL-8 secretion in monocytes and macrophages. The inhibition of IL- 8 secretion with different doses of lutein was not significant. The analysis of IL-8 indicated that different doses of lutein could reduce 


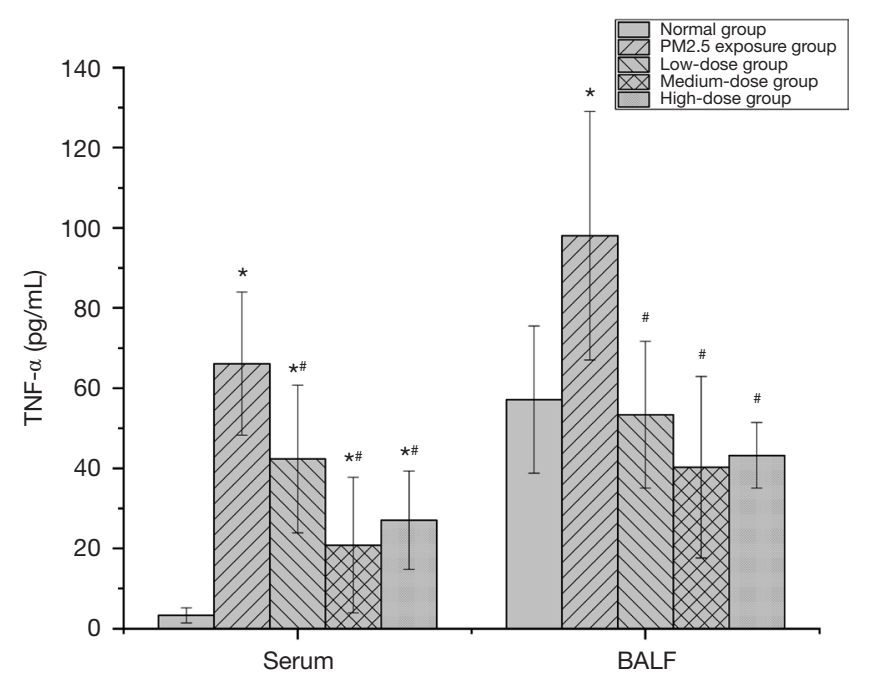

Figure 4 The effect of lutein on TNF- $\alpha$ in serum and BALF. *, compared with the normal group, there was a significant difference $(\mathrm{P}<0.05)$; ${ }^{\text {, }}$ compared with the $\mathrm{PM} 2.5$ exposure group, there was a significant difference $(\mathrm{P}<0.05)$.

the accumulation of the inflammatory cytokine IL- 8 in lung tissues, thus alleviating the accumulation of inflammatory cytokines and neutrophils in lung tissues caused by PM2.5 exposure and reducing the degree of the inflammatory reaction in lung tissue.

\section{The effect of PM2.5 on TNF- $\alpha$ and the intervention \\ effect of lutein}

TNF- $\alpha$ is the inflammatory cytokine involved earliest in the inflammatory response. It is an indicator that reflects the degree of cellular inflammatory action and can activate mononuclear macrophages, neutrophils, and vascular endothelial cells, resulting in the release of a large number of inflammatory cytokines and inflammatory tissue injury. The serum TNF- $\alpha$ level in rats was detected, and the results are shown in Figure 4. Compared with that in the normal group, the TNF- $\alpha$ levels in the serum and BALF of rats in the PM2.5 exposure group dramatically increased, indicating that PM2.5 caused serious inflammatory effects. After the administration of different doses of lutein, the TNF- $\alpha$ levels in the serum and BALF of rats both significantly decreased $(\mathrm{P}<0.05)$, and the degrees of reduction in TNF- $\alpha$ in the medium- and high-dose groups were higher than that in the low-dose group. These results indicated that lutein alleviated the release of the proinflammatory cytokine TNF- $\alpha$ induced by PM2.5.

\section{The effect of PM2.5 on cell damage in rat lung tissues and the antagonizing effect of lutein}

The effects of PM2.5 exposure and pretreatment with different doses of lutein on the activities of LDH, ACP, and AKP in the BALF of rats were investigated. As shown in Figure 5A, compared with that in the normal group, PM2.5 exposure caused an increase in the LDH activity in BALF from rats; however, the difference was not significant $(\mathrm{P}>0.05)$. Although the LDH activity in BALF from rats after different doses of lutein intervention decreased, the differences were not significant $(\mathrm{P}>0.05)$. As shown in Figure $5 B$, compared with that in the normal group, the ACP activity in BALF from rats in the PM2.5 exposure group significantly increased $(\mathrm{P}<0.05)$; compared with that in the PM2.5 exposure group, ACP activity in the BALF from rats in the low-, medium-, and high-dose lutein groups significantly decreased $(\mathrm{P}<0.05)$. As shown in Figure 5 C, compared with that in the normal group, the AKP activity in BALF from rats in the PM2.5 exposure group significantly increased $(\mathrm{P}<0.05)$; compared with that in the PM2.5 exposure group, AKP activity in the BALF from rats in the low-, medium-, and high-dose lutein groups all significantly decreased $(\mathrm{P}<0.05)$. These results indicated that PM2.5 could cause substantial damage to cells in the lung tissues of rats to some extent and that lutein uptake could antagonize the toxic effect of PM2.5 on rat lung tissue.

\section{Discussion}

PM2.5 is a mixture with complex components. With differences in cities, seasons, and time, its composition and hazards also vary. PM2.5 enters the blood system through the lung barrier. Long-term exposure has direct damaging effects on lung tissue. Cohort studies showed that for every $10 \mu \mathrm{g} / \mathrm{m}^{3}$ increase in PM2.5, the overall mortality rate for the population, the incidence of cardiopulmonary disease, and the incidence of lung cancer increased by $4 \%, 6 \%$, and $8 \%$, respectively (15). Animal studies (16-22) indicated that the percentage of inflammatory cells increased as a result of oxidative damage and the inflammatory response, that the levels of MDA, NOS, AKP, and ACP increased, and that the levels of inflammation-related cytokines IL-6, IL-8, and TNF- $\alpha$ increased. This study combined the above sensitive indicators to establish a PM2.5 oxidative damage model. The results showed that after exposure to $10 \mathrm{mg} / \mathrm{kg}$ PM2.5 continuously for 7 days, the levels of damage 

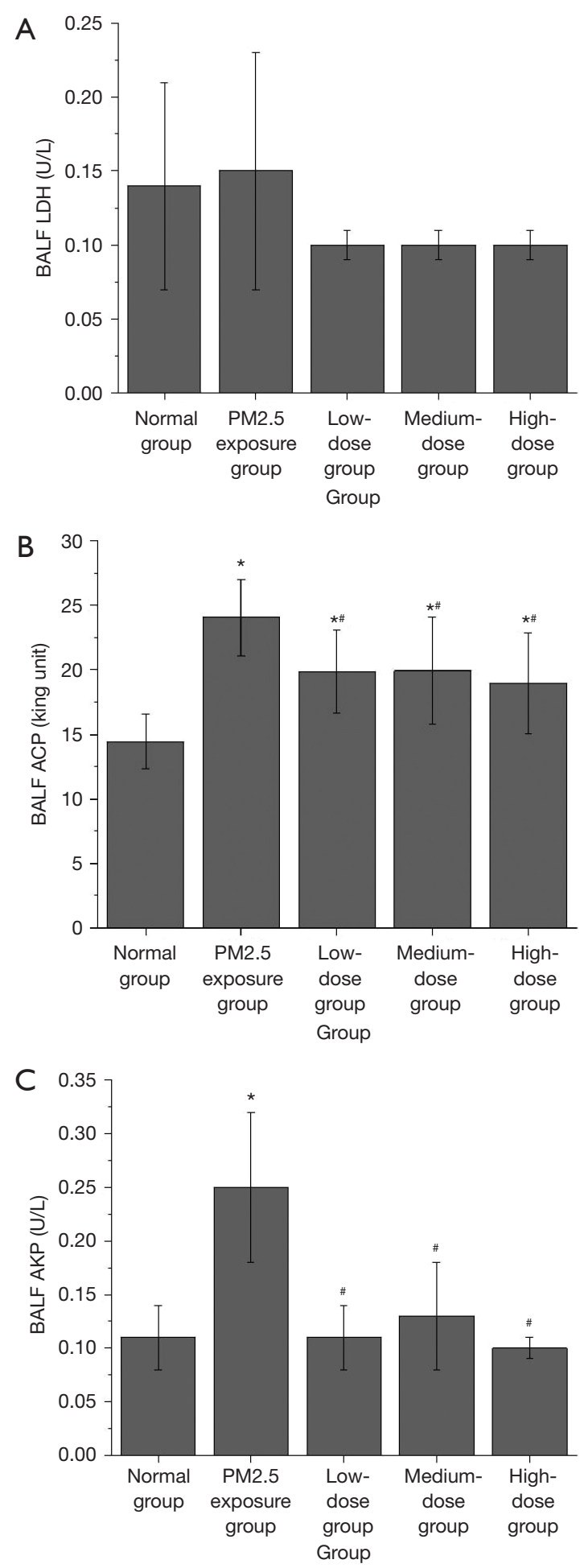

Figure 5 The antagonizing effect of lutein on the toxic effect of PM2.5 in lung tissues. (A,B,C) Are LDH, ACP, and AKP activity, respectively. *, compared with the normal group, there was a significant difference $(\mathrm{P}<0.05) ;{ }^{*}$, compared with the $\mathrm{PM} 2.5$ exposure group, there was a significant difference $(\mathrm{P}<0.05)$. indicators, including MDA, NOS, 8-OHdG, ACP, AKP, IL-6, IL-8, and TNF- $\alpha$ all significantly increased, whereas the level of the antioxidant indicator GSH decreased. These results indicated that PM2.5 caused oxidative damage and inflammatory symptoms in lung tissue. Therefore, a PM2.5 damage model was established using the above method.

The hazards caused by PM2.5 have received widespread attention. Management measures for PM2.5 health hazards have also gradually improved. It is very necessary to perform studies on effective health protection measures for the management of air pollution. Previous studies on the function of lutein mainly focused on visual fatigue and the liver. For example, lutein can decrease the lipid oxidation marker MDA and the level of DNA oxidation biomarker 8 -OHdG in the retina and improve oxidative damage in retinal tissue (10,23). In liver tissue (24-30), lutein can increase the activity of $\mathrm{NA}^{+}-\mathrm{K}^{+}$-ATPase and mitochondrial $\mathrm{Ca}^{2+}$-ATPase, increase the levels of SOD and GSH, and decrease the activities of TNOS and iNOS and the level of $\mathrm{NO}$; in addition, it can increase HO-1 expression and downregulate TLR4 mRNA expression to exert its antioxidative stress function. These results indicate that lutein, in different tissues, has excellent biological activities such as anti-inflammatory and antioxidative functions. Therefore, this study performed animal experiments to observe the damaging effect of PM2.5 in rat lung tissues and validate the antagonizing effect of lutein on PM2.5 in lung tissues. The analysis of serum MDA, SOD, GSH, NOS, and 8-OHdG showed that PM2.5 increased the oxidative damage indicators MDA and NOS, increased the DNA damage indicator8-OHdG, and decreased GSH; after the administration of lutein, oxidative damage was relieved. The analysis of inflammatory cytokines IL-6, IL-8, and TNF- $\alpha$ showed that PM2.5 significantly promoted the expression of pro-inflammatory cytokines to increase the development of inflammation in lung tissues. Lutein reduced the secretion level of inflammatory cytokines to alleviate the development of inflammation. LDH, ACP, and AKP directly reflect the degree of cell damage. After PM2.5 exposure, cell membrane permeability decreases after cell membrane damage, and large amounts of LDH, ACP, and AKP are released. Lutein intervention can significantly alleviate cell damage caused by PM2.5 to maintain cell membrane permeability.

This study confirmed that lutein in lung tissues antagonized the respiratory tract injury caused by PM2.5. Its protective mechanism was directly associated with its antioxidant and anti-inflammatory effects to alleviate direct 
cell damage resulting from PM2.5. The investigation of PM2.5 damage and mechanism of antagonism to further implement interventional measures targeting different components of PM2.5 can provide basic data for the prevention of health hazards caused by particles and for the development of lutein-related products.

\section{Acknowledgments}

Funding: The work is supported by the National Key Research and Development Program of the Ministry of Science and Technology of the People's Republic of China (No. 2016YFD0400601).

\section{Footnote}

Reporting Checklist: The authors have completed the ARRIVE reporting checklist. Available at http://dx.doi. org/10.21037/apm-20-1138

Data Sharing Statement: Available at http://dx.doi. org/10.21037/apm-20-1138

Conflicts of Interest: All authors have completed the ICMJE uniform disclosure form (available at http://dx.doi. org/10.21037/apm-20-1138). The authors report grants from Ministry of Science and Technology of the People's Republic of China, during the conduct of the study.

Ethical Statement: The authors are accountable for all aspects of the work in ensuring that questions related to the accuracy or integrity of any part of the work are appropriately investigated and resolved. The study was approved by The Animal Care \&Welfare Committee Institute of Materia Medica, Chinese Academy of Medical Sciences \& Peking Union Medical College (ID: 00005303), and is in compliance with its guidelines for the care and use of animals.

Open Access Statement: This is an Open Access article distributed in accordance with the Creative Commons Attribution-NonCommercial-NoDerivs 4.0 International License (CC BY-NC-ND 4.0), which permits the noncommercial replication and distribution of the article with the strict proviso that no changes or edits are made and the original work is properly cited (including links to both the formal publication through the relevant DOI and the license). See: https://creativecommons.org/licenses/by-nc-nd/4.0/.

\section{References}

1. Deng X, Zhang F, Rui W, et al. PM2.5-induced oxidative stress triggers autophagy in human lung epithelial A549 cells. Toxicol In Vitro 2013;27:1762-70.

2. Gualtieri M, Longhin E, Mattioli M, et al. Gene expression profiling of A549 cells exposed to Milan PM2.5. Toxicol Lett 2012;209:136-45.

3. Tian CY, Yan M, Zhang Y, et al. Effect of particulate matter 2.5 at urban centre of Hangzhou on lung impairment in rats. Zhongguo Ying Yong Sheng Li Xue Za Zhi 2018;34:299-303.

4. Etchie TO, Etchie AT, Adewuyi GO, et al. The gains in life expectancy by ambient PM2.5 pollution reductions in localities in Nigeria. Environ Pollut 2018;236:146-57.

5. Song $\mathrm{C}, \mathrm{He} \mathrm{J}, \mathrm{Wu} \mathrm{L}$, et al. Health burden attributable to ambient PM 2.5 in China. Environ Pollut 2017;223:575-86.

6. Wang $\mathrm{Z}$, Lin $\mathrm{X}$. The determination and contents of lutein, zeaxanthin and $\beta$-carotene in vegetables in Beijing region. Acta Nutrimenta Sinica 2010;32:290-4.

7. Trevithick-Sutton CC, Foote CS, Collins M, et al. The retinal carotenoids zeaxanthin and lutein scavenge superoxide and hydroxyl radicals: a chemiluminescence and ESR study. Mol Vis 2006;12:1127-35.

8. Bhosale P, Serban B, Bernstein PS. Retinal carotenoids can attenuate formation of $\mathrm{A} 2 \mathrm{E}$ in the retinal pigment epithelium. Arch Biochem Biophys 2009;483:175-81.

9. Bian Q, Gao S, Zhou J, et al. Lutein and zeaxanthin supplementation reduces photooxidative damage and modulates the expression of inflammation-related genes in retinal pigment epithelial cells. Free Radic Biol Med 2012;53:1298-307.

10. Deng Q, Zang X, Chen P, et al. Protective effect of plant polysaccharide extracts combined with lutein on visible light-induced retinal damage via an antioxidant mechanism. Food Science 2017;38:233-8.

11. Olmedilla-Alonso B, Beltrán-de-Miguel B, EstévezSantiago R, et al. Markers of lutein and zeaxanthin status in two age groups of men and women: dietary intake, serum concentrations, lipid profile and macular pigment optical density. Nutr J 2014;13:52.

12. Hunter CA, Jones SA. IL-6 as a keystone cytokine in health and disease. Nat Immunol 2015;16:448-57.

13. Jones BS, Jerome MS, Miley D, et al. Pilot phase II study of metronomic chemotherapy in combination with bevacizumab in patients with advanced non-squamous non-small cell lung cancer. Lung Cancer 2017;106:125-30.

14. Liu Z, Xu S, Xiao N, et al. Overexpression of IL-8 and 
MMP-9 confer high malignant phenotype in patients with non-small cell lung cancer. Zhongguo Fei Ai Za Zhi 2010;13:795-802.

15. Vinikoor-Imler LC, Davis JA, Luben TJ. An ecologic analysis of country-level PM2.5 concentrations and LUNG cancer incidence and mortality. Int J Environ Res Public Health 2011;8:1865-71.

16. Li LZ, Yuan XY, Wang YM, et al. PM2.5 induces oxidative damage and affects nuclear factor-erythroid 2 related factor 2 pathway in human umbilical vein endothelial cells. Zhonghua Yu Fang Yi Xue Za Zhi 2016;50:710-5.

17. Liu H, Wu Y, Chen M, et al. Characteristics of atmospheric PM2.5 in Nanjing City and its cytotoxicity in human lung epithelial cells A549. Research of Environmental Sciences 2018;31:1736-42.

18. He J, Wang Y, Zhao Z, et al. Oxidative damage related to PM2.5 exposure in human embryonic stem cell-derived fibroblasts. Chinese Journal of Preventive Medicine 2016;50:705-9.

19. He J, Zhao Y, Huang J, et al. Study on the lung damage in mice induced by a combined exposure of PM2.5 and formaldehyde and its molecular mechanism. Asian Journal of Ecotoxicology 2018;13:87-93.

20. Cheng Z, Liu Y, Qi X, et al. Influence of PM2.5 and PM10 exposure on physiological and pathological parameters of rats under different polluted weather in Beijing. Asian Journal of Ecotoxicology 2018;13:226-40.

21. Guo L, Zhang K, Liu J, et al. Mechanism of lung injury caused by high temperature and PM2.5 in COPD rats. Journal of Environmental \& Occupational Medicine
2018;35:898-904.

22. Kim Y; Seo JH; Kim H. $\beta$-Carotene and lutein inhibit hydrogen peroxide-induced activation of NF-kappaB and IL-8 expression in gastric epithelial AGS cells. J Nutr Sci Vitaminol (Tokyo) 2011;57:216-23.

23. Hong Y, Fang L, Zheng Y, et al. Effects of Cordyceps militaris extracts on lung injury induced by PM2.5 in mice. Food Research and Development 2019;40:36-41.

24. Niu M, Li S, Niu Q, et al. Preventive effects of lutein on liver toxicity in mice induced by arsenic. Journal of Hygiene Research 2015;(4):615-9.

25. Zhang H, Wang J, Bai J, et al. Protective effect of lutein on acute alcoholic liver injury in mice. Pharmacology and Clinics of Chinese Materia Medica 2012;28:69-72.

26. Zhang H, Liu S, Bai J, et al. The Effect of lutein pretreatment on cisplatin-induced hepatic ultrastructure damage in rats. Tianjin Medical Journal 2012;(5):490-2.

27. Liu S, Liu Y, Zhang H, et al. Protective effect of lutein on acute hepatotoxicity induced by $\mathrm{N}, \mathrm{N}$-dimethylformamide in mice. Pharmacology and Clinics of Chinese Materia Medica 2011;27:26-8.

28. Pei L. Study on protective effect of lutein on acute chemical liver injury in mice. Chinese Journal of Gerontology 2009;(6):685-7.

29. Zhang X, Liu H, Jin J, et al. Study on lutein protective on acute chemical hepatic injury of mice. Food Science and Technology 2009;34:79-82.

30. Pei L, Hui B, Dong F. Effect of Tagetes erecta L.'s extraction on antioxidative function in aged rats. Chinese Journal of Gerontology 2007;(9):814-7.
Cite this article as: Chen X, Chen Y, Chen Y, Wang X, He M. Protective effect of lutein on oxidative stress damage caused by acute PM2.5 exposure in rats. Ann Palliat Med 2020;9(4):20282036. doi: 10.21037/apm-20-1138 